\title{
The structure of $0^{+}$states in ${ }^{16} \mathrm{O}$ using real-time evolution method
}

\author{
Hideaki Motoki ${ }^{1, *}$ and Masaaki Kimura ${ }^{1,2,3,4, * *}$ \\ ${ }^{1}$ Department of Physics, Hokkaido University, Sapporo 060-0810, Japan \\ ${ }^{2}$ Nuclear Reaction Data Centre (JCPRG), Hokkaido University, Sapporo 060-0810, Japan \\ ${ }^{3}$ Research Center for Nuclear Physics (RCNP), Osaka University, Ibaraki 567-0047, Japan \\ ${ }^{4}$ RIKEN (The Institute of Physical and Chemical Research), Wako, Saitama 351-0198, Japan
}

\begin{abstract}
C}$ clustering in ${ }^{16} \mathrm{O}$ has been vigorously studied. In the 2000s, a new picture was proposed that the Hoyle state, ${ }^{12} \mathrm{C}\left(0_{2}^{+}\right)$, is a Bose-Einstein condensate of three $\alpha$ particles by the so-called THSR framework. As a next step, many researchers are interested in $4 \alpha$ condensate state in ${ }^{16} \mathrm{O}$. In this work, a microscopic calculation named the real-time evolution method (REM) was first applied to a $4 \alpha$ system. As a result, the $0^{+}$states in ${ }^{16} \mathrm{O}$ up to $4 \alpha$ condensate state were expected to be reproduced simultaneously for the first time.
\end{abstract}

\section{Introduction}

At the beginning of this century, a new picture was proposed that the Hoyle state is regarded as the Bose-Einstein condensate of three $\alpha$ particles. It provides us a unique opportunity to study the condensate of bosons composed of four fermions. Up to now, the $3 \alpha$ condensate is almost established, and thus we focus on the $4 \alpha$ system. Several works for ${ }^{16} \mathrm{O}, 4 \alpha$ Orthogonality Condition Model (OCM) [1], and extended Tohsaki-Horiuchi-Schuck-Röpke (eTHSR) [2] have been performed. In these studies, the $0_{6}^{+}$state was theoretically concluded as a candidate of the $4 \alpha$ condensate $[1,2]$. However, the former treats an $\alpha$ particle as a boson and the latter imposes the restrictions on the symmetry of the system resulting in the lack of $0_{5}^{+}$state in the $4 \alpha$ OCM. Therefore we employ a microscopic model, REM [3].

\section{Theoretical Framework}

The Hamiltonian for the $N \alpha$ systems composed of $4 N$ nucleons is the same as in the original paper of the REM [3] except the effective nucleon-nucleon 2(3)-body interaction. For evaluating our method with others, we used the Tohsaki No. 1 effective nucleon-nucleon interaction [4]. The intrinsic wave function of the $N \alpha$ system is defined as

$$
\Phi\left(\boldsymbol{Z}_{1}, \cdots, \boldsymbol{Z}_{N}\right)=\mathcal{A}\left\{\Phi_{\alpha}\left(\boldsymbol{Z}_{1}\right) \cdots \Phi_{\alpha}\left(\boldsymbol{Z}_{N}\right)\right\}
$$

where $\mathcal{A}$ represents the antisymmetrization operator. The $\Phi_{\alpha}(\boldsymbol{Z})$ denotes the wave packet of the $\alpha$ cluster located at $Z$. The normalized GCM wave function of ${ }^{16} \mathrm{O}, \Psi^{0^{+}} \mathrm{O}$, is defined as

\footnotetext{
*e-mail: motoki@nucl.sci.hokudai.ac.jp

**e-mail: masaaki@nucl.sci.hokudai.ac.jp
} 


$$
\Psi_{16}^{0^{+} \mathrm{O}}=\sum_{i}\left\{f_{i} \hat{P}^{0^{+}} \Phi_{i}\left(\boldsymbol{Z}_{1}, \cdots, \boldsymbol{Z}_{4}\right)+g_{i} \hat{P}^{0^{+}} \Phi_{i}\left(\boldsymbol{Z}_{1}^{*}, \cdots, \boldsymbol{Z}_{4}^{*}\right)\right\}
$$

where $\hat{P}^{0^{+}}$is the angular-momentum $(J)$ and the parity $(\pi)$ projection operator for $J^{\pi}=0^{+}$. In the REM, the position and momentum of $\alpha$ particles are governed by the equation-of-motion (EOM) of the Gaussian wave packets. In principle, the $\Psi_{{ }^{0} \mathrm{O}_{\mathrm{O}}^{+}}$can be divided into the $\alpha+{ }^{12} \mathrm{C}$ component $\Psi_{\alpha+{ }^{12} \mathrm{C}}^{0^{+}}$and the residual part $\Psi_{\text {res }}^{0^{+}}$. The squared amplitude $|w|^{2}$, where $w$ is the coefficient of the $\Psi_{\alpha+{ }^{12} \mathrm{C}}^{0^{+}}$, can be estimated using the method in the Ref. [5].

\section{Results}

Results were obtained as follows: The intrinsic excitation energy and rebound radius in the REM are $40 \mathrm{MeV}$ and $16.0 \mathrm{fm}$, respectively. The time of the EOM was evolved until 6000 $\mathrm{fm} / c$ by $6 \mathrm{fm} / c$ units. We used the $r^{2}$-constraint method [6]. The cutoff value is $7.0 \mathrm{fm}$.

We first discuss the excitation energy spectra measured from the $4 \alpha$ threshold and r.m.s. charge radii compared with the eTHSR [2] in Fig. 1 . As for the bound states, $0_{1}^{+}$and $0_{2}^{+}$, the energies are slightly lower than that of the eTHSR reflecting the size of model space. Table 2 shows the calculated and observed r.m.s. radii and monopole matrix elements from the $0_{1}^{+}$ state. The monopoles can be understood as an enhancement of the clustering in the excited states. Considering the consistency of the radius and monopole with the OCM, the $0_{6}^{+}$state in the REM is expected to be a corresponding state of $0_{5}^{+}$state in the OCM. Note that the $0_{5}^{+}$state in the OCM is mainly composed of $\alpha+{ }^{12} \mathrm{C}\left(1^{-}\right)$which is not described in the eTHSR due to the size of the model space. The $0_{V}^{+}$state in the eTHSR is said to be a $4 \alpha$ condensate. As for the $0_{7}^{+}$state in the REM, though the radius is almost consistent with that of the eTHSR, the monopole is much larger than that of the eTHSR, which imply that the $0_{7}^{+}$state in the REM is a candidate of $4 \alpha$ condensate and has a characteristic property of the clustering enhancement.

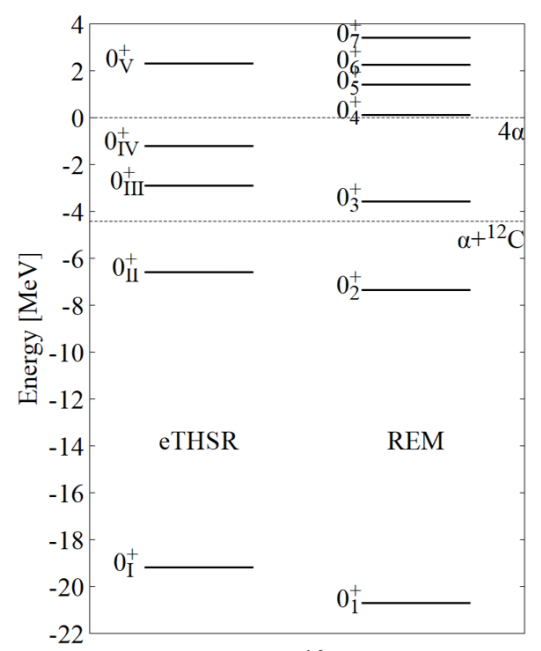

Figure 1. Energy spectra of ${ }^{16} \mathrm{O}\left(0^{+}\right)$states measured from the $4 \alpha$ threshold of the eTHSR [2] and REM.
Table 1. $\alpha+{ }^{12} \mathrm{C}\left(0_{1}^{+}\right)$components $|w|^{2}$, the maximized overlaps in Eq. (3) and the main components.

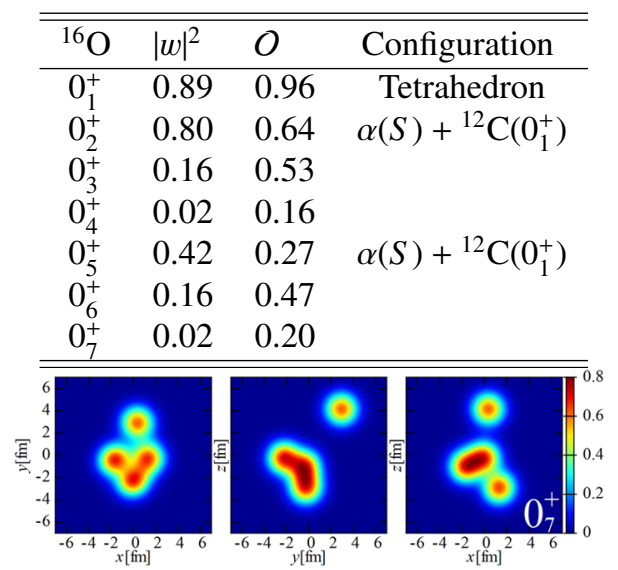

Figure 2. Density distribution of the intrinsic wave function maximized for $0_{7}^{+}$state using Eq. (3).

We estimated the squared amplitudes of $\alpha+{ }^{12} \mathrm{C}\left(0_{1}^{+}\right)$components $|w|^{2}$, which is shown in Table 1 . The ${ }^{12} \mathrm{C}\left(0_{1}^{+}\right)$state was made by the same process explained in the original paper of the REM [3]. The maximized overlaps defined in Eq. (3) for each ${ }^{16} \mathrm{O}\left(0^{+}\right)$state and the configurations are also shown in Table 1.

$$
O=\left|\left\langle\Psi_{16}^{0^{+}} \mid P^{0^{+}} \Phi_{\mathrm{opt}}\right\rangle\right|^{2} /\left\langle P^{0^{+}} \Phi_{\mathrm{opt}} \mid P^{0^{+}} \Phi_{\mathrm{opt}}\right\rangle .
$$


Table 2. r.m.s. charge radii $R_{\mathrm{rms}}$ and monopole matrix elements from the ground state $\mathcal{M}\left(E 0 ; 0_{1}^{+} \rightarrow 0_{f}^{+}\right)$of the experiment [7] and the $4 \alpha$ OCM [1], eTHSR [2], and REM calculations in ${ }^{16} \mathrm{O}$.

\begin{tabular}{|c|c|c|c|c|c|c|c|c|}
\hline \multirow[b]{2}{*}{ REM } & \multicolumn{4}{|c|}{$R_{\mathrm{rms}}[\mathrm{fm}]$} & \multicolumn{4}{|c|}{$\mathcal{M}\left(E 0 ; 0_{1}^{+} \rightarrow 0_{f}^{+}\right)\left[e \mathrm{fm}^{2}\right]$} \\
\hline & EXP. & OCM & eTHSR & REM & EXP. & OCM & eTHSR & REM \\
\hline $0_{1}^{+}$ & $2.71(0.02)$ & 2.7 & 2.7 & 2.7 & & & & \\
\hline $0_{2}^{+}$ & & 3.0 & 3.2 & 3.1 & 3.55 & 3.9 & 5.9 & 5.8 \\
\hline $0_{3}^{+}$ & & 3.1 & 3.3 & 3.2 & 4.03 & 2.4 & 5.7 & 4.3 \\
\hline $0_{4}^{+}$ & & & & 5.7 & & & & 0.9 \\
\hline $0_{5}^{+}$ & & 4.0 & 4.9 & 5.2 & & 2.4 & 0.8 & 1.1 \\
\hline $0_{6}^{+}$ & & 3.1 & & 3.1 & 3.3 & 2.6 & & 2.9 \\
\hline $0_{7}^{+}$ & & 5.6 & 4.9 & 4.8 & & 1.0 & 0.7 & 2.4 \\
\hline
\end{tabular}

It is easily understood that the $0_{1}^{+}$state has a large squared amplitude and the overlap resulting from the tetrahedral configuration. The $0_{2,5}^{+}$states have also a rather large squared amplitude, which means that these states are composed of $\alpha+{ }^{12} \mathrm{C}\left(0_{1}^{+}\right)$. This is consistent with the $4 \alpha$ OCM [1] in which they say that the $0_{4}^{+}$state in the $4 \alpha$ OCM is the higher nodal state of the $0_{2}^{+}$state. Although the $0_{3,6}^{+}$states have overlaps about $50 \%$, the squared amplitude of the $\alpha+{ }^{12} \mathrm{C}\left(0_{1}^{+}\right)$component is small, which implies that these states are composed of the other types of configurations; i.g. $\alpha+{ }^{12} \mathrm{C}\left(2^{+}\right), \alpha+{ }^{12} \mathrm{C}\left(1^{-}\right)$. Note that the $0_{4}^{+}$state is expected as the continuum state due to the quite large radius. It is expected that the $4 \alpha$ condensate is composed of $\alpha+{ }^{12} \mathrm{C}\left(0_{2}^{+}\right)$resulting in the small squared amplitude of the $\alpha+{ }^{12} \mathrm{C}\left(0_{1}^{+}\right)$ component and small overlap. Therefore the $0_{7}^{+}$state is a candidate of the $4 \alpha$ condensate. The density distribution of the intrinsic wave function maximized for the $0_{7}^{+}$state is shown in Fig. 2. It looks that The four $\alpha$ particles are loosely interacting with each other.

\section{Summary}

We focused on the $0^{+}$states in ${ }^{16} \mathrm{O}$ to investigate the $4 \alpha$ condensate state by adopting the REM analyzing the excitation energy, r.m.s. charge radius and monopole matrix element. Additionally, we showed the squared amplitude of the $\alpha+{ }^{12} \mathrm{C}\left(0_{1}^{+}\right)$component As a result, a candidate of the $4 \alpha$ condensate state was obtained. The ${ }^{16} \mathrm{O}\left(0^{+}\right)$states up to the $4 \alpha$ condensate state were expected to be microscopically described simultaneously for the first time. We will calculate the $\alpha+{ }^{12} \mathrm{C}\left(0_{2}^{+}\right)$component to conclude the $0_{7}^{+}$state as the $4 \alpha$ condensate state.

\section{Acknowledgments}

The numerical calculations were carried out on the high performance computing server at RCNP in Osaka University and on XC40 at YITP in Kyoto University.

\section{References}

[1] Y. Funaki, T. Yamada, H. Horiuchi, G. Röpke, P. Schuck, A. Tohsaki, Phys. Rev. Lett. 101, 082502 (2008)

[2] Y. Funaki, Phys. Rev. C 97, 021304 (2018)

[3] R. Imai, T. Tada, M. Kimura, Phys. Rev. C 99, 064327 (2019)

[4] A. Tohsaki, Phys. Rev. C 49, 1814 (1994)

[5] M. Kimura, Phys. Rev. C 69, 044319 (2004)

[6] Y. Funaki, H. Horiuchi, A. Tohsaki, Progress of Theoretical Physics 115, 115 (2006)

[7] F. Ajzenberg-Selove, Nuclear Physics A 460, 1 (1986) 